\title{
MATRIX FUNCTIONS THAT PRESERVE THE STRONG PERRON-FROBENIUS PROPERTY*
}

\author{
PIETRO PAPARELLA ${ }^{\dagger}$
}

\begin{abstract}
In this note, we characterize matrix functions that preserve the strong PerronFrobenius property using the real Jordan canonical form of a real matrix.
\end{abstract}

Key words. Matrix function, Real Jordan Canonical form, Perron-Frobenius Theorem, Eventually Positive Matrix

AMS subject classifications. 15A16, 15B48, 15A21.

1. Introduction. A real matrix has the Perron-Frobenius property if its spectral radius is a positive eigenvalue corresponding to an entrywise nonnegative eigenvector. The strong Perron-Frobenius property further requires that the spectral radius is simple; that it dominates in modulus every other eigenvalue; and that it has an entrywise positive eigenvector.

In [17, Micchelli and Willoughby characterized matrix functions that preserve doubly nonnegative matrices. In [7, Guillot et al. used these results to solve the critical exponent conjecture established in [12. In [1, Bharali and Holtz characterized entire functions that preserve nonnegative matrices of a fixed order and, in addition, they characterized matrix functions that preserve nonnegative block triangular, circulant, and symmetric matrices. In [4, Elhashash and Szyld characterized entire functions that preserve sets of generalized nonnegative matrices.

In this work, using the characterization of a matrix function via the real Jordan canonical form established in [16, we characterize matrix functions that preserve the strong Perron-Frobenius property. Although our results are similar to those presented in 4, the assumption of entirety of a function is dropped in favor of analyticity in some domain containing the spectrum of a matrix.

2. Notation. Denote by $M_{n}(\mathbb{C})$ (respectively, $M_{n}(\mathbb{R})$ ) the algebra of complex (respectively, real) $n \times n$ matrices. Given $A \in M_{n}(\mathbb{C})$, the spectrum of $A$ is denoted by $\sigma(A)$, and the spectral radius of $A$ is denoted by $\rho(A)$.

\footnotetext{
$*$

${ }^{\dagger}$ Division of Engineering and Mathematics, University of Washington Bothell, Bothell, Washington 98011, USA (pietrop@uw.edu)
} 
The direct sum of the matrices $A_{1}, \ldots, A_{k}$, where $A_{i} \in M_{n_{i}}(\mathbb{C})$, denoted by $A_{1} \oplus \cdots \oplus A_{k}, \bigoplus_{i=1}^{k} A_{i}$, or $\operatorname{diag}\left(A_{1}, \ldots, A_{k}\right)$, is the $n \times n$ matrix

$$
\left[\begin{array}{ccc}
A_{1} & & \\
& \ddots & \\
& & A_{k}
\end{array}\right], n=\sum_{i=1}^{k} n_{i} .
$$

For $\lambda \in \mathbb{C}, J_{n}(\lambda)$ denotes the $n \times n$ Jordan block with eigenvalue $\lambda$. For $A \in$ $M_{n}(\mathbb{C})$, denote by $J=Z^{-1} A Z=\bigoplus_{i=1}^{t} J_{n_{i}}\left(\lambda_{i}\right)=\bigoplus_{i=1}^{t} J_{n_{i}}$, where $\sum n_{i}=n$, a Jordan canonical form of $A$. Denote by $\lambda_{1}, \ldots, \lambda_{s}$ the distinct eigenvalues of $A$, and, for $i=1, \ldots, s$, let $m_{i}$ denote the index of $\lambda_{i}$, i.e., the size of the largest Jordan block associated with $\lambda_{i}$. Denote by $\mathrm{i}$ the imaginary unit, i.e., $\mathrm{i}:=\sqrt{-1}$.

A domain $\mathcal{D}$ is any open and connected subset of $\mathbb{C}$. We call a domain selfconjugate if $\bar{\lambda} \in \mathcal{D}$ whenever $\lambda \in \mathcal{D}$ (i.e., $\mathcal{D}$ is symmetric with respect to the real-axis). Given that an open and connected set is also path-connected, it follows that if $\mathcal{D}$ is self-conjugate, then $\mathbb{R} \cap \mathcal{D} \neq \emptyset$.

3. Background. Although there are multiple ways to define a matrix function (see, e.g., 9]), our preference is via the Jordan Canonical Form.

Definition 3.1. Let $f: \mathbb{C} \longrightarrow \mathbb{C}$ be a function and denote by $f^{(j)}$ the $j$ th derivative of $f$. The function $f$ is said to be defined on the spectrum of $A$ if the values

$$
f^{(j)}\left(\lambda_{i}\right), \quad j=0, \ldots, m_{i}-1, \quad i=1, \ldots, s,
$$

called the values of the function $f$ on the spectrum of $A$, exist.

Definition 3.2 (Matrix function via Jordan canonical form). If $f$ is defined on the spectrum of $A \in M_{n}(\mathbb{C})$, then

$$
f(A):=Z f(J) Z^{-1}=Z\left(\bigoplus_{i=1}^{t} f\left(J_{n_{i}}\right)\right) Z^{-1},
$$

where

$$
f\left(J_{n_{i}}\right):=\left[\begin{array}{cccc}
f\left(\lambda_{i}\right) & f^{\prime}\left(\lambda_{i}\right) & \ldots & \frac{f^{\left(n_{i}-1\right)}\left(\lambda_{i}\right)}{\left(n_{i}-1\right) !} \\
& f\left(\lambda_{i}\right) & \ddots & \vdots \\
& & \ddots & f^{\prime}\left(\lambda_{i}\right) \\
& & & f\left(\lambda_{i}\right)
\end{array}\right] .
$$


The following theorem is well-known (for details see, e.g., 11], 14]; for a complete proof, see, e.g., [6]).

Theorem 3.3 (Real Jordan canonical form). If $A \in M_{n}(\mathbb{R})$ has $r$ real eigenvalues (including multiplicities) and c complex conjugate pairs of eigenvalues (including multiplicities), then there exists an invertible matrix $R \in M_{n}(\mathbb{R})$ such that

$$
R^{-1} A R=\left[\begin{array}{ll}
\bigoplus_{k=1}^{r} J_{n_{k}}\left(\lambda_{k}\right) & \\
& \bigoplus_{k=r+1}^{r+c} C_{n_{k}}\left(\lambda_{k}\right)
\end{array}\right]
$$

where:

1.

$$
C_{j}(\lambda):=\left[\begin{array}{cccc}
C(\lambda) & I_{2} & & \\
& C(\lambda) & \ddots & \\
& & \ddots & I_{2} \\
& & & C(\lambda)
\end{array}\right] \in M_{2 j}(\mathbb{R})
$$

2.

$$
C(\lambda):=\left[\begin{array}{cc}
\Re(\lambda) & \Im(\lambda) \\
-\Im(\lambda) & \Re(\lambda)
\end{array}\right] \in M_{2}(\mathbb{R}) ;
$$

3. $\Im\left(\lambda_{k}\right)=0, k=1, \ldots, r$; and

4. $\Im\left(\lambda_{k}\right) \neq 0, k=r+1, \ldots, r+c$.

Proposition 3.4 ([16, Corollary 2.11]). Let $\lambda \in \mathbb{C}, \lambda \neq 0$, and let $f$ be a function defined on the spectrum of $J_{k}(\lambda) \oplus J_{k}(\bar{\lambda})$. For $j$ a nonnegative integer, let $f_{\lambda}^{(j)}$ denote $f^{(j)}(\lambda)$. If $C_{k}(\lambda)$ and $C(\lambda)$ are defined as in (3.3) and (3.4), respectively, then

$$
f\left(C_{k}(\lambda)\right)=\left[\begin{array}{cccc}
f\left(C_{\lambda}\right) & f^{\prime}\left(C_{\lambda}\right) & \ldots & \frac{f^{(k-1)}\left(C_{\lambda}\right)}{(k-1) !} \\
& f\left(C_{\lambda}\right) & \ddots & \vdots \\
& & \ddots & f^{\prime}\left(C_{\lambda}\right) \\
& & & f\left(C_{\lambda}\right)
\end{array}\right] \in M_{2 k}(\mathbb{C})
$$

and, moreover,

$$
f\left(C_{k}(\lambda)\right)=\left[\begin{array}{cccc}
C\left(f_{\lambda}\right) & C\left(f_{\lambda}^{\prime}\right) & \ldots & C\left(\frac{f_{\lambda}^{(k-1)}}{(k-1) !}\right) \\
& C\left(f_{\lambda}\right) & \ddots & \vdots \\
& & \ddots & C\left(f_{\lambda}^{\prime}\right) \\
& & & C\left(f_{\lambda}\right)
\end{array}\right] \in M_{2 k}(\mathbb{R})
$$


if and only if $\overline{f_{\lambda}^{(j)}}=f_{\bar{\lambda}}^{(j)}$.

We recall the Perron-Frobenius theorem for positive matrices (see [11, Theorem 8.2.11]).

Theorem 3.5. If $A \in M_{n}(\mathbb{R})$ is positive, then

(i) $\rho:=\rho(A)>0$;

(ii) $\rho \in \sigma(A)$;

(iii) there exists a positive vector $x$ such that $A x=\rho x$;

(iv) $\rho$ is a simple eigenvalue of $A$; and

(v) $|\lambda|<\rho$ for every $\lambda \in \sigma(A)$ such that $\lambda \neq \rho$.

One can verify that the matrix

$$
B=\left[\begin{array}{cc}
2 & 1 \\
2 & -1
\end{array}\right]
$$

satisfies properties (i) through (v) of Theorem 3.5. but obviously contains a negative entry. This motivates the following concept.

Definition 3.6. A matrix $A \in M_{n}(\mathbb{R})$ is said to possess the strong PerronFrobenius property if $A$ satisfies properties (i) through (v) of Theorem 3.5.

It can also be shown that the matrix $B$ given in (3.5) satisfies $B^{k}>0$ for $k \geq 4$, which leads to the following generalization of positive matrices.

Definition 3.7. A matrix $A \in M_{n}(\mathbb{R})$ is eventually positive if there exists a nonnegative integer $p$ such that $A^{k}>0$ for all $k \geq p$.

The following theorem relates the strong Perron-Frobenius property with eventually positive matrices (see [8, Lemma 2.1], [13, Theorem 1], or [18, Theorem 2.2]).

Theorem 3.8. A real matrix $A$ is eventually positive if and only if $A$ and $A^{\top}$ possess the strong Perron-Frobenius property.

4. Main Results. Before we state our main results, we begin with the following definition.

Definition 4.1. A function $f: \mathbb{C} \longrightarrow \mathbb{C}$ defined on a self-conjugate domain $\mathcal{D}$, $\mathcal{D} \cap \mathbb{R}^{+} \neq \emptyset$, is called Frobenius 1 if

(i) $\overline{f(\lambda)}=f(\bar{\lambda}), \lambda \in \mathcal{D}$;

(ii) $|f(\lambda)|<f(\rho)$, whenever $|\lambda|<\rho$, and $\lambda, \rho \in \mathcal{D}$.

\footnotetext{
${ }^{1}$ We use the term 'Frobenius' given that such a function preserves Frobenius multi-sets, introduced by Friedland in [5].
} 
Remark 4.2. Condition (i) implies $f(r) \in \mathbb{R}$, whenever $r \in \mathcal{D} \cap \mathbb{R}$; and condition (ii) implies $f(r) \in \mathbb{R}^{+}$, whenever $r \in \mathcal{D} \cap \mathbb{R}^{+}$.

The following theorem is our first main result.

Theorem 4.3. Let $A \in M_{n}(\mathbb{R})$ and suppose that $A$ is diagonalizable and possesses the strong Perron-Frobenius property. If $f: \mathbb{C} \longrightarrow \mathbb{C}$ is a function defined on the spectrum of $A$, then $f(A)$ possesses the strong Perron-Frobenius property if and only if $f$ is Frobenius.

Proof. Suppose that $f$ is Frobenius. For convenience, denote by $f_{\lambda}$ the scalar $f(\lambda)$. Following Theorem 3.3 and Proposition 3.4 the matrix

$$
f(A)=R\left[\begin{array}{lll}
f_{\rho(A)} & & \\
& \bigoplus_{k=2}^{r} f_{\lambda_{k}} & \\
& & \bigoplus_{k=r+1}^{r+c} C\left(f_{\lambda_{k}}\right)
\end{array}\right] R^{-1}
$$

where $R=\left[\begin{array}{ll}x & R^{\prime}\end{array}\right], x>0$, is real. If $\sigma(A)=\left\{\rho(A), \lambda_{2}, \ldots, \lambda_{n}\right\}$, then $\sigma(f(A))=$ $\left\{f_{\rho(A)}, f_{\lambda_{2}}, \ldots, f_{\lambda_{n}}\right\}$ (see, e.g., [9][Theorem 1.13(d)]) and because $f$ is Frobenius, it follows that $\left|f_{\lambda_{k}}\right|<f_{\rho(A)}$ for $k=2, \ldots, n$. Moreover, from (4.1) it follows that $f(A) x=f_{\rho(A)} x$. Thus, $f(A)$ possesses the strong Perron-Frobenius property.

Conversely, if $f$ is not Frobenius, then the matrix $f(A)$, given by (4.1), is not real (e.g, $\exists \lambda \in \sigma(A), \lambda \in \mathbb{R}$ such that $f(\lambda) \notin \mathbb{R}$ ), or $f(A)$ does not retain the strong Perron-Frobenius property (e.g., $\exists \lambda \in \sigma(A)$ such that $|f(\lambda)| \geq f(\rho(A)))$.

EXAMPLE 4.4. Table 4.1 lists examples of Frobenius functions for diagonalizable matrices that possess the strong Perron-Frobenius property.

\begin{tabular}{|c|c|}
\hline$f$ & $\mathcal{D}$ \\
\hline$f(z)=z^{p}, p \in \mathbb{N}$ & $\mathbb{C}$ \\
\hline$f(z)=|z|$ & $\mathbb{C}$ \\
\hline$f(z)=z^{1 / p}, p \in \mathbb{N}, p$ even & $\left\{z \in \mathbb{C}: z \notin \mathbb{R}^{-}\right\}$ \\
\hline$f(z)=z^{1 / p}, p \in \mathbb{N}, p$ odd, $p>1$ & $\mathbb{C}$ \\
\hline$f(z)=\sum_{k=0}^{n} a_{k} z^{k}, a_{k}>0$ & $\mathbb{C}$ \\
\hline$f(z)=\exp (z)$ & $\mathbb{C}$ \\
\hline
\end{tabular}

For matrices that are not diagonalizable, i.e., possessing Jordan blocks of size two or greater, given (3.1) it is reasonable to assume that $f$ is complex-differentiable, i.e., analytic. We note the following result, which is well known (see, e.g., [2], 3], 10, 
Theorem 3.2], [15], or [19]).

TheOrem 4.5 (Reflection Principle). Let $f$ be analytic in a self-conjugate domain $\mathcal{D}$ and suppose that $I:=\mathcal{D} \cap \mathbb{R} \neq \emptyset$. Then $\overline{f(\lambda)}=f(\bar{\lambda})$ for every $\lambda \in \mathcal{D}$ if and only if $f(r) \in \mathbb{R}$ for all $r \in I$.

The Reflection Principle leads immediately to the following result.

Corollary 4.6. An analytic function $f: \mathbb{C} \longrightarrow \mathbb{C}$ defined on a self-conjugate domain $\mathcal{D}, \mathcal{D} \cap \mathbb{R}^{+} \neq \emptyset$, is Frobenius if and only if

(i) $f(r) \in \mathbb{R}$, whenever $r \in \mathcal{D} \cap \mathbb{R}$; and

(ii) $|f(\lambda)|<f(\rho)$, whenever $|\lambda|<\rho$ and $\lambda, \rho \in \mathcal{D}$.

Lemma 4.7. Let $f$ be analytic in a domain $\mathcal{D}$ and suppose that $I:=\mathcal{D} \cap \mathbb{R} \neq \emptyset$. If $f(r) \in \mathbb{R}$ for all $r \in I$, then $f^{(j)}(r) \in \mathbb{R}$ for all $r \in I$ and $j \in \mathbb{N}$..

Proof. Proceed by induction on $j$ : when $j=1$, note that, since $f$ is analytic on $\mathcal{D}$, it is holomorphic (i.e., complex-differentiable) on $\mathcal{D}$. Thus,

$$
f^{\prime}(r):=\lim _{z \rightarrow r} \frac{f(z)-f(r)}{z-r}
$$

exists for all $z \in \mathcal{D}$; in particular,

$$
f^{\prime}(r)=\lim _{x \rightarrow r} \frac{f(x)-f(r)}{x-r}, x \in I,
$$

and the conclusion that $f^{\prime}(r) \in \mathbb{R}$ follows by the hypothesis that $f(x) \in \mathbb{R}$ for all $x \in I$.

Next, assume that the result holds when $j=k-1>1$. As above, note that $f^{(k)}(r)$ exists and

$$
f^{(k)}(r)=\lim _{x \rightarrow r} \frac{f^{(k-1)}(x)-f^{(k-1)}(r)}{x-r}, x \in I
$$

so that $f^{(k)}(r) \in \mathbb{R}$.

Theorem 4.8. Let $A \in M_{n}(\mathbb{R})$ and suppose that $A$ possesses the strong PerronFrobenius property. If $f: \mathbb{C} \longrightarrow \mathbb{C}$ is an analytic function defined in a self-conjugate domain $\mathcal{D}$ containing $\sigma(A)$, then $f(A)$ possesses the strong Perron-Frobenius property if and only if $f$ is Frobenius.

Proof. Suppose that $f$ is Frobenius. Following Theorem 3.3, there exists an invertible matrix $R$ such that

$$
R^{-1} A R=\left[\begin{array}{lll}
\rho(A) & & \\
& \bigoplus_{k=2}^{r} J_{n_{k}}\left(\lambda_{k}\right) & \\
& & \bigoplus_{k=r+1}^{r+c} C_{n_{k}}\left(\lambda_{k}\right)
\end{array}\right],
$$


where

$$
R=\left[\begin{array}{ll}
x & R^{\prime}
\end{array}\right], x>0 .
$$

Because $f$ is Frobenius, following Theorem 4.5, $\overline{f(\lambda)}=f(\bar{\lambda})$ for all $\lambda \in \mathcal{D}$. Since $f$ is analytic, $f^{(j)}$ is analytic for all $j \in \mathbb{N}$ and, following Lemma $4.7 f^{(j)}(r) \in \mathbb{R}$ for all $r \in I$. Another application of Theorem 4.5 yields that $\overline{f^{(j)}(\lambda)}=f^{(j)}(\bar{\lambda})$ for all $\lambda \in \mathcal{D}$. Hence, following Proposition 3.4 the matrix

$$
f(A)=R\left[\begin{array}{lll}
f(\rho(A)) & & \\
& \bigoplus_{k=2}^{r} f\left(J_{n_{k}}\left(\lambda_{k}\right)\right) & \\
& & \bigoplus_{k=r+1}^{r+c} f\left(C_{n_{k}}\left(\lambda_{k}\right)\right)
\end{array}\right] R^{-1}
$$

is real and possesses the strong Perron-Frobenius property.

The proof of the converse is identical to the proof of the converse of Theorem 4.3.

Corollary 4.9. Let $A \in M_{n}(\mathbb{R})$ and suppose that $A$ is eventually positive. If $f: \mathbb{C} \longrightarrow \mathbb{C}$ is an analytic function defined in a self-conjugate domain $\mathcal{D}$ containing $\sigma(A)$, then $f(A)$ is eventually positive if and only if $f$ is Frobenius.

Proof. Follows from Theorem 4.8 and the fact that $f\left(A^{\top}\right)=(f(A))^{\top}(\underline{9}$, Theorem 1.13(b)]).

REMARK 4.10. Aside from the function $f(z)=|z|$, which is nowhere differentiable, every function listed in Table 4.1 is analytic and Frobenius.

5. Acknowledgements. I gratefully acknowledge Hyunchul Park for discussions arising from this research and the anonymous referees for their helpful suggestions.

\section{REFERENCES}

[1] G. Bharali and O. Holtz. Functions preserving nonnegativity of matrices. SIAM J. Matrix Anal. Appl., 30(1):84-101, 2008.

[2] J. W. Brown and R. V. Churchill. Complex Variables and Applications. McGraw-Hill Science/Engineering/Math, ninth edition, 2013.

[3] J. B. Conway. Functions of one complex variable. II, volume 159 of Graduate Texts in Mathematics. Springer-Verlag, New York, 1995.

[4] A. Elhashash and D. B. Szyld. Matrix functions preserving sets of generalized nonnegative matrices. Electron. J. Linear Algebra, 20:673-690, 2010.

[5] S. Friedland. On an inverse problem for nonnegative and eventually nonnegative matrices. Israel J. Math., 29(1):43-60, 1978.

[6] I. Gohberg, P. Lancaster, and L. Rodman. Invariant subspaces of matrices with applications. Canadian Mathematical Society Series of Monographs and Advanced Texts. John Wiley \& Sons, Inc., New York, 1986. A Wiley-Interscience Publication. 
[7] D. Guillot, A. Khare, and B. Rajaratnam. The critical exponent conjecture for powers of doubly nonnegative matrices. Linear Algebra Appl., 439(8):2422-2427, 2013.

[8] D. Handelman. Positive matrices and dimension groups affiliated to $C^{*}$-algebras and topological Markov chains. J. Operator Theory, 6(1):55-74, 1981.

[9] N. J. Higham. Functions of matrices. Society for Industrial and Applied Mathematics (SIAM), Philadelphia, PA, 2008. Theory and computation.

[10] N. J. Higham, D. S. Mackey, N. Mackey, and F. Tisseur. Functions preserving matrix groups and iterations for the matrix square root. SIAM J. Matrix Anal. Appl., 26(3):849-877 (electronic), 2005.

[11] R. A. Horn and C. R. Johnson. Matrix analysis. Cambridge University Press, Cambridge, 1990. Corrected reprint of the 1985 original.

[12] C. R. Johnson, B. Lins, and O. Walch. The critical exponent for continuous conventional powers of doubly nonnegative matrices. Linear Algebra Appl., 435(9):2175-2182, 2011.

[13] C. R. Johnson and P. Tarazaga. On matrices with Perron-Frobenius properties and some negative entries. Positivity, 8(4):327-338, 2004.

[14] P. Lancaster and M. Tismenetsky. The theory of matrices. Computer Science and Applied Mathematics. Academic Press Inc., Orlando, FL, second edition, 1985.

[15] S. Lang. Complex analysis, volume 103 of Graduate Texts in Mathematics. Springer-Verlag, New York, fourth edition, 1999.

[16] J. J. McDonald, P. Paparella, and M. J. Tsatsomeros. Matrix roots of eventually positive matrices. Linear Algebra Appl., 456:122-137, 2014.

[17] C. A. Micchelli and R. A. Willoughby. On functions which preserve the class of Stieltjes matrices. Linear Algebra Appl., 23:141-156, 1979.

[18] D. Noutsos. On Perron-Frobenius property of matrices having some negative entries. Linear Algebra Appl., 412(2-3):132-153, 2006.

[19] W. Rudin. Real and complex analysis. McGraw-Hill Book Co., New York, third edition, 1987. 